\title{
Theory of the Mercury's spin-orbit motion and analysis of its main librations
}

\begin{abstract}
N. Rambaux and E. Bois
Observatoire Aquitain des Sciences de l'Univers, Université Bordeaux 1, UMR CNRS/INSU 5804, BP 89, 33270 Floirac, France

Received 16 June 2003 / Accepted 13 August 2003

Abstract. The 3:2 spin-orbit resonance between the rotational and orbital motions of Mercury (the periods are $P_{\phi}=56.646$ and $P_{\lambda}=87.969$ days respectively) results from a functional dependance of the tidal friction adding to a non-zero eccentricity and a permanent asymmetry in the equatorial plane of the planet. The upcoming space missions, MESSENGER and BepiColombo with onboard instrumentation capable of measuring the rotational parameters stimulate the objective to reach an accurate theory of the rotational motion of Mercury. For obtaining the real motion of Mercury, we have used our BJV model of solar system integration including the coupled spin-orbit motion of the Moon. This model, expanded in a relativistic framework, had been previously built in accordance with the requirements of the Lunar Laser Ranging observational accuracy. We have extended the BJV model by generalizing the spin-orbit couplings to the terrestrial planets (Mercury, Venus, Earth, and Mars). The updated model is called SONYR (acronym of Spin-Orbit $N$-BodY Relativistic model). As a consequence, the SONYR model gives an accurate simultaneous integration of the spin-orbit motion of Mercury. It permits one to analyze the different families of rotational librations and identify their causes such as planetary interactions or the parameters involved in the dynamical figure of the planet. The spin-orbit motion of Mercury is characterized by two proper frequencies (namely $\Phi=15.847 \mathrm{yrs}$ and $\Psi=1066 \mathrm{yrs}$ ) and its 3:2 resonance presents a second synchronism which can be understood as a spin-orbit secular resonance $(\Pi=278898 \mathrm{yrs}$ ). A new determination of the mean obliquity is proposed in the paper. By using the SONYR model, we find a mean obliquity of 1.6 arcmin. This value is consistent with the Cassini state of Mercury. Besides, we identify in the Hermean librations the impact of the uncertainty of the greatest principal moment of inertia $\left(C / M R^{2}\right)$ on the obliquity and on the libration in longitude ( 2.3 milliarcsec and 0.45 arcsec respectively for an increase of $1 \%$ on the $C / M R^{2}$ value). These determinations prove to be suitable for providing constraints on the internal structure of Mercury.
\end{abstract}

Key words. methods: numerical - celestial mechanics - planets and satellites: individual: Mercury

\section{Introduction}

Before 1965, the rotational motion of Mercury was assumed to be synchronous with its orbital motion. In 1965, Pettengill \& Dyce discovered a 3:2 spin-orbit resonance state by using Earth-based radar observations (the Mercury's rotation period is $P_{\phi}=58.646$ days while the orbital one is $P_{\lambda}=87.969$ days). This surprising resonance results from a non-zero eccentricity and a permanent asymmetry in the equatorial plane of the planet. In addition, the 3:2 resonance strongly depends on the functional dependance of the tidal torque on the rate of the libration in longitude. Moreover the 3:2 resonance state is preserved by the tidal torque (Colombo \& Shapiro 1966). The main dynamical features of Mercury have been established during the 1960s in some pioneer works such as Colombo (1965), Colombo \& Shapiro (1966), Goldreich \& Peale (1966) and Peale (1969). Goldreich \& Peale (1966) notably studied the probability of resonance capture and showed that the $3: 2$ ratio is the only possible one for a significant probability of capture. In addition, in a tidally evolved system, the spin pole is

Send offprint requests to: $\mathrm{N}$. Rambaux, e-mail: rambaux@obs.u-bordeaux1.fr expected to be trapped in a Cassini state (Colombo 1966; Peale 1969, 1973). The orbital and rotational parameters are indeed matched in such a way that the spin pole, the orbit pole, and the solar system invariable pole remain coplanar while the spin and orbital poles precess. The reader may find in Balogh \& Campieri (2002) a review report on the present knowledge of Mercury whose the interest is nowadays renewed by two upcoming missions: MESSENGER (NASA, Solomon et al. 2001) and BepiColombo (ESA, ISAS, Anselin \& Scoon 2001).

Our work deals with the physical and dynamical causes that contribute to induce librations around an equilibrium state defined by a Cassini state. In order to wholly analyze the spin-orbit motion of Mercury and its rotational librations, we used a gravitational model of the solar system including the Moon's spin-orbit motion. The framework of the model has been previously constructed by Bois, Journet \& Vokrouhlický (BJV model) in accordance with the requirements of Lunar Laser Ranging (LLR thereafter) observational accuracy (see for instance a review report by Bois 2000). The approach of the model consists in integrating the $N$-body problem on the basis of the gravitation description given by the Einstein's general relativity theory according to a formalism derived from the 
first post-Newtonian approximation level. The model is solved by modular numerical integration and controlled in function of the different physical contributions and parameters taken into account. We have extended this model to the integration of the rotational motions of the terrestrial planets (Mercury, Venus, Earth, and Mars) including their spin-orbit couplings. The updated model is then called SONYR (acronym of Spin-Orbit $N$ bodY Relativistic model). As a consequence, using SONYR, the $N$-body problem for the solar system and the spin motion of Mercury are simultaneously integrated. Consequently we may analyze and identify the different families of Hermean rotational librations with the choice of the contributions at our disposal.

Starting with the basic spin-orbit problem according to Goldreich \& Peale (1966), we have computed a surface of section for the Mercury's rotation showing its very regular behavior. We have calculated again the proper frequency for the spin-orbit resonance state of Mercury. Using our model, an important part of the present study deals with the main perturbations acting on the spin-orbit motion of Mercury such as the gravitational figure of the planet as well as the planetary effects and their hierarchy. A detailed analysis of the resulting rotational librations due to these effects is presented and described in the paper. A new determination of the Hermean mean obliquity is also proposed. Moreover, we identify in the Hermean librations the impact of the variation of the greatest principal moment of inertia on the instantaneous obliquity and on the libration in longitude. Such a signature gives noticeable constraints on the internal structure of Mercury.

\section{Geometry of the spin-orbit coupling problem}

According to Goldreich \& Peale (1966), we consider the spinorbit motion of Mercury with its spin axis normal to the orbital plane. The orbit is assumed to be fixed and unvariable (semimajor axis $a$ and its eccentricity $e$ ). The position of Mercury is determined by its instantaneous radius $r$ while its rotational orientation is specified by the angle $\theta$. The orbital longitude is specified by the true anomaly $f$ while the angle $\theta-f$ measures the angle between the axis of least moment of inertia of Mercury and the Sun-to-Mercury line (see Fig. 1). According to these assumptions, the dynamical problem of the spin-orbit motion of Mercury is reduced to a one-dimensional pendulumlike equation as follows:

$C \ddot{\theta}+\frac{3}{2}(B-A) \frac{G M_{\odot}}{r^{3}} \sin 2(\theta-f)=0$

where $G$ is the gravitational constant, $M_{\odot}$ the solar mass, and $A, B$, and $C$ the principal moments of inertia of Mercury. This equation of motion has only a single degree of freedom for the spin-orbit coupling, the characteristic angle of rotation $\theta$, but depends explicitly on time through the distance $r$ to the planet and the non-uniform Keplerian motion of the true anomaly $f$. As a consequence, it is a problem not reducible by quadrature and Eq. (1) is non-integrable (Wisdom 1987).

In order to know the structure of the phase space of the Mercury's rotation, a surface of section for its spin-orbit coupling is very useful. Let be $\alpha=\sqrt{\frac{3(B-A)}{C}}$ the asphericity of the

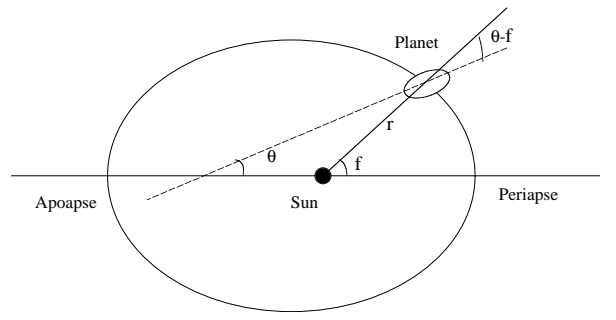

Fig. 1. Geometry of the spin-orbit coupling problem. $\boldsymbol{r}$ is the radius vector, $f$ the true anomaly and $\theta$ is the characteristic angle of rotation.

Mercury's dynamical figure combining the principal moments of inertia $A, B$, and $C$. Equation (1) becomes:

$\ddot{\theta}+\frac{1}{2} n^{2}\left(\frac{a}{r}\right)^{3} \alpha^{2} \sin 2(\theta-f)=0$

where $n$ is the orbital mean motion, $f$ and $\theta$ as defined above. The numerical integration of Eq. (2) yields to Fig. 2 showing stroboscopically, i.e. once point per orbit at the periapse, various trajectories of the phase portrait of Mercury $(\theta$ and $1 / n \mathrm{~d} \theta / \mathrm{d} t$ on the axes $x$ and $y$ respectively). Figure 2 a illustrates the different quasiperiodic librations around the 1:2, 1:1 (synchronous), $3: 2$, and 2:1 rotation states while Fig. $2 \mathrm{~b}$ shows a zoom of the present 3:2 resonance state for Mercury. Let us notice that in the 1:2 rotation state, the two libration areas are shifted by $\pi / 2$ with respect to the other rotation states. The former rotation rate of Mercury was higher and the planet could have experienced large and chaotic variations in obliquity at some time of the past (Laskar \& Robutel 1993). Due to its slowing down by tidal despin, the Mercury's rotation has then encountered different resonance states, from higher orders to the one trapped in the present state. Goldreich \& Peale (1966) have shown that this present state (the 3:2 resonance) was the first one with a substantial probability of capture. This one indeed depends crucially on the functional dependence of the tidal torque acting on the spin-orbit motion. This dependence is expressed by the variation rate of the longitude libration angle $\gamma$ as defined below. In the end, the stabilization of Mercury at the 3:2 spin-orbit resonance is due to permanent asymmetry on the equatorial plane, as well as its non-zero eccentricity equal to 0.206 (Colombo 1966; Colombo \& Shapiro 1966).

According to the Chirikov resonance overlap criterion (1979), the chaotic behavior appears when the asphericity of the body is larger than the following critical value:

$\alpha^{\mathrm{cr}}=\frac{1}{2+\sqrt{14 e}}$

where $e$ is the orbital eccentricity. In the case of Mercury, $\alpha=0.0187$ is lower than the critical value $\alpha^{\text {cr }}=0.2701$. As a consequence, the spin-orbit behavior is regular. The zoom in Fig. $2 b$ shows indeed that the separatrix surrounding the $3: 2$ resonance state is very small. The width of the associated chaotic zone is then estimated to the order of $10^{-43}$ (Wisdom et al. 1984). Consequently, tidal friction pulls Mercury accross the chaotic separatrix in a single libration period.

From Eq. (1), it is possible to obtain an integrable approximated equation using the spin-orbit resonance, the spin rate $\dot{\theta}$ being commensurable with the mean orbital motion $n$. 


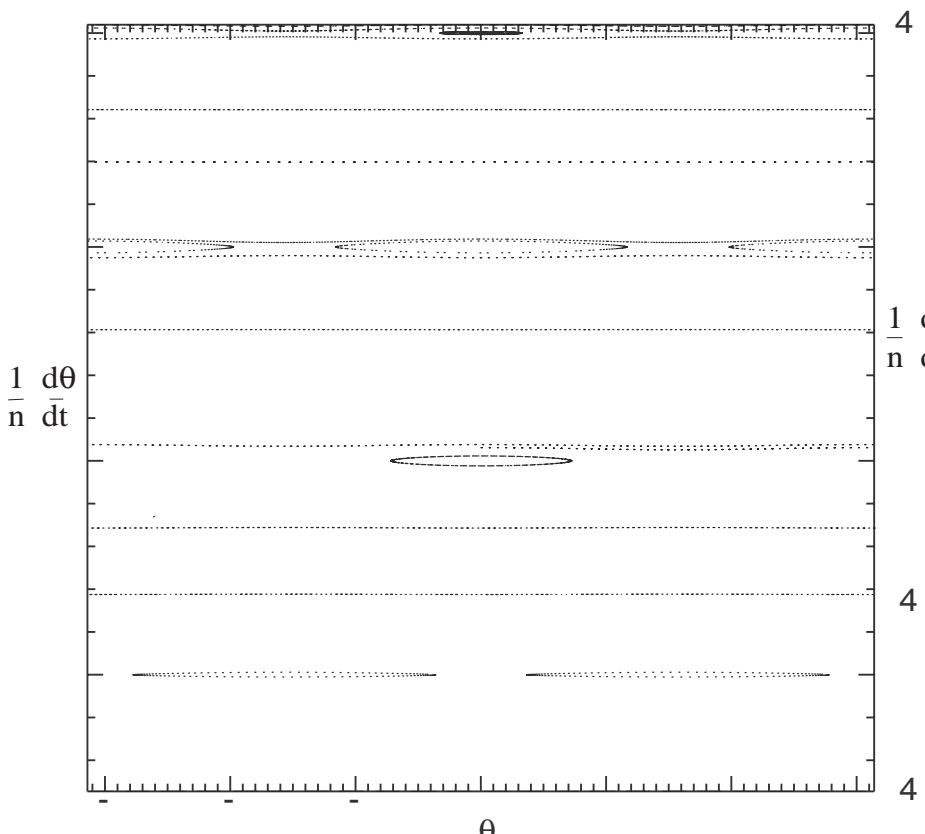

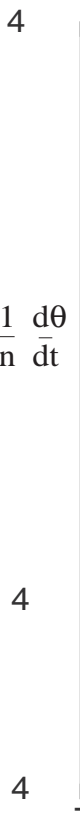

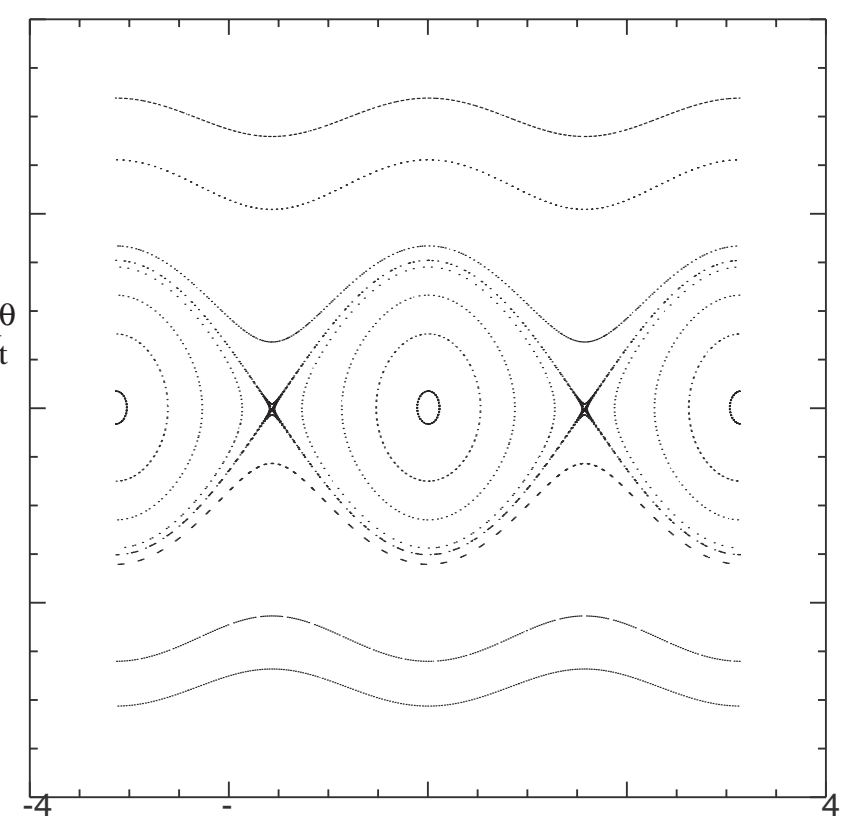

$\theta$

Fig. 2. Surface of section of the Mercury's spin-orbit coupling ( $\alpha=0.0187, e=0.206$ ). The rate of change of the orientation is plotted versus the orientation of Mercury defined by the angle $\theta$ at every pericenter passage. The spin axis is constrained to be normal to the orbital plane. The plot a) makes in evidence the different quasiperiodic librations around the 1:2, 1:1 (synchronous), 3:2, and 2:1 rotation states. The plot b) is a zoom of the real 3:2 resonance state of Mercury. The chaotic zone is microscopic and non visible at this scale.

Following Murray \& Dermott (2000), by introducing a new variable $\gamma=\theta-p n$ where $p$ parametrizes the resonance ratio ( $p=\frac{3}{2}$ in the case of Mercury), one may expand the equation in form-like Poisson series. Taking into account that $\dot{\gamma} \ll n$, one averages all the terms over one orbital period, and finally obtain the following equation:

$\ddot{\gamma}+\frac{3}{2} n^{2} \frac{B-A}{C} H(p, e) \sin 2 \gamma=0$

where $H(p, e)$ is a power series in eccentricity. In the case of Mercury, this expression is written as follows:

$H\left(\frac{3}{2}, e\right)=\frac{7}{2} e-\frac{123}{16} e^{3}$.

Finally, the proper frequency of the Mercury's spin-orbit motion is:

$\omega_{0}=n\left[3 \frac{B-A}{C}|H(p, e)|\right]^{\frac{1}{2}}$

which by using the values listed in Table 2 gives the proper period of $15.830 \mathrm{yrs}$.

Balogh \& Giamperi (2002) developed Eq. (2) and obtained the following expression:

$\ddot{\gamma}+\alpha_{0} \sum_{q} G_{20 q}(e) \sin [2 \gamma+(1-q) M]=0$

where the $G_{20 q}$ coefficients are eccentricity functions defined by Kaula (1966). The $G_{201}$ coefficient is equal to $H\left(\frac{3}{2}, e\right)$. $\alpha_{0}=\frac{3}{2} \frac{B-A}{C}=1.76 \times 10^{-4}$ for the Mercury case. Figure 3 presents then the numerical solution of the formula (7) (thanks

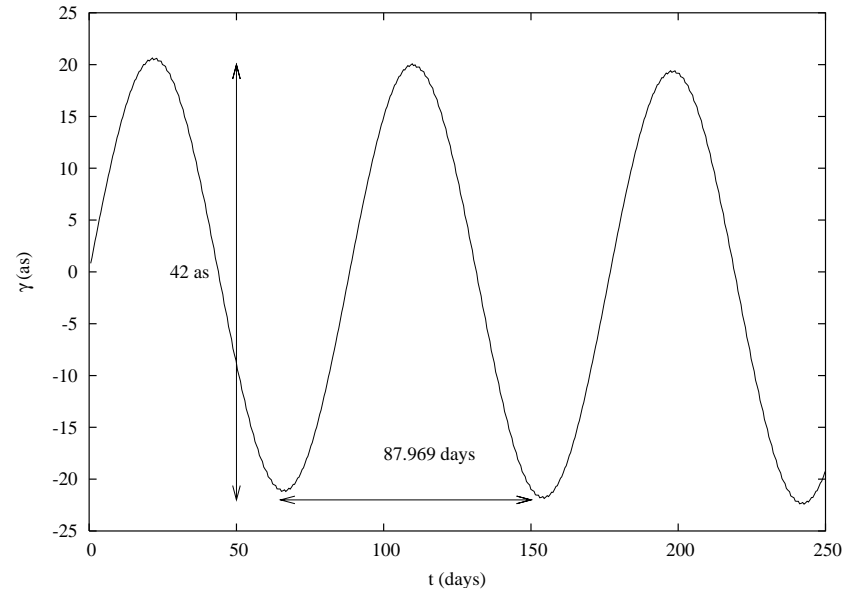

Fig. 3. The spin-orbit solution of Mercury in the planar case (Eq. (7)) plotted over 250 days. Arcseconds are on the vertical axis and days on the horizontal axis. Short-term librations have a period of 87.969 days (the orbital period of Mercury) and 42 as of amplitude.

to Giampieri, private communication). The angle $\gamma$ describes a periodic behavior with a period equal to the revolution of Mercury. The amplitude of $42 \operatorname{arcsec}$ (as) depends on the value of $\alpha_{0}$. The behavior of the $\gamma$ angle is nicely matched by the approximate formula of Jhen \& Corral (2003):

$\gamma=\phi_{0} \sin M+\phi_{1} \sin 2 M$

where $\phi_{0}=\alpha_{0}\left(G_{200}-G_{202}\right)$ and $\phi_{1}=\frac{\alpha_{0}}{4}\left(G_{20-1}-G_{203}\right)$. The authors noticed that the ratio of the two amplitudes $\left(\phi_{0}\right.$ and $\left.\phi_{1}\right)$ does not depend on the $\alpha_{0}$ parameter, and $K=\frac{\phi_{0}}{\phi_{1}}=-9.483$. 
However, the above equations describe a spin-orbit motion of Mercury where the spin axis is normal to the orbital plane while the orbital motion is Keplerian.

\section{The extended BJV model: SONYR}

\subsection{Theory}

In order to wholly analyze the spin-orbit motion of Mercury and its rotational librations, we have enlarged a gravitational model (called BJV) of the solar system including the Moon's spin-orbit motion. The accurate theory of the Moon's spin-orbit motion, related to this BJV model, was constructed by Bois, Journet \& Vokrouhlický in accordance with the high accuracy of the LLR observations (see previous papers: Bois et al. 1992; Bois \& Journet 1993; Bois \& Vokrouhlický 1995; Bois et al. 1996; Bois \& Girard 1999). The approach of the BJV model consists in integrating the $N$-body problem (including translational and rotational motions) on the basis of the gravitation description given by the Einstein's general relativity theory. The equations have been developped in the DSX formalism presented in a series of papers by Damour et al. (Damour et al. 1991, 1992, 1993, 1994). For purposes of celestial mechanics, to our knowledge, it is the most suitable formulation of the post-Newtonian (PN) theory of motion for a system of $N$ arbitrary extended, weakly self-graviting, rotating and deformable bodies in mutual interactions. The DSX formalism, derived from the first post-Newtonian approximation level, gives the post-Newtonian representation of the translational motions of the bodies as well as their rotational ones with respect to the locally transported frames with the bodies.

Gravitational fields of the extended bodies are parameterized in multipole moment expansions: $\left(M_{L}^{\mathrm{A}}, S_{L}^{\mathrm{A}}\right)$ define the mass and spin Blanchet-Damour multipoles characterizing the PN gravitational field of the extended bodies while $\left(G_{L}^{\mathrm{A}}, H_{L}^{\mathrm{A}}\right)$ are tidal gravitoelectric and gravitomagnetic PN fields. Because we do not dispose of dynamical equations for the quadrupole moments $M_{\mathrm{ab}}^{\mathrm{A}}$, and although the notion of rigidity faces conceptual problems in the theory of relativity, we have adopted the "rigid-multipole" model of the extended bodies as known from the Newtonian approach. Practically this is acceptable since the relativistic quadrupole contributions are very small. Consequently and because it is conventional in geodynamical research to use spherical harmonics analysis of the gravitational fields with the corresponding notion of harmonic coefficients $\left(C_{1 \mathrm{~m}}^{\mathrm{A}}, S_{1 \mathrm{~m}}^{\mathrm{A}}\right)$, the quadrupole moments $M_{\mathrm{ab}}^{\mathrm{A}}$ have been expressed in those terms, according to reasons and assumptions given in Bois \& Vokrouhlický (1995). Gravitational figures as well as the figure-figure interactions of the bodies are then represented by expansions in spherical harmonics (Borderies 1978; Shutz 1981). Moreover, internal structures of solid deformable bodies, homogeneous or with core-mantle interfaces, are represented by several terms and parameters arising from tidal deformations of the bodies (both elastic and anelastic). More details and references on these topics are given in the above quoted papers related to our works concerning the theory of the Moon's spin-orbit motion.
The BJV model, as described above, has been extended to the spin-orbit integration of the terrestrial planets (Mercury, Venus, Earth, and Mars). This new model is henceforth called SONYR (for Spin-Orbit $N$-bodY Relativistic model). In the present paper framework, the SONYR model is devoted to the detailed analysis of the complete spin-orbit motion of Mercury.

The simultaneous integration of the solar system, including the Mercury's spin-orbit motion, uses a global reference system given by the solar system barycenter. Nevertheless, let us recall that local dynamically non-rotating frames show a slow (de Sitter) rotation with respect to the kinematically non-rotating frames. As a consequence, the reference frame for the Mercury's rotation is affected by a slow precession of its axes transported with the translational motion of Mercury. In the Earth's case, the de Sitter secular precession of the Earth reference frame is close to 1.92 as/cy (see Fukushima 1991; Bizouard et al. 1992; Bois \& Vokrouhlický 1995). Consequently, the real rotation of Mercury has not to be expressed in an inertial system fixed in space, but in a local dynamically non-rotating frame fallen down in the gravitational field of the Sun. Because of the proximity of Mercury to the Sun, its de Sitter precession may be expected quite significant.

In the end, the SONYR model and its analysis method take into account (i) the experience in post-Newtonian gravitation in the definition of reference frames required to deal with rotational motions combined with translational ones, and (ii) the modern knowledge of dynamical systems for studiing librations as quasi-periodic solutions according to the axiomatic presented in Bois (1995). We can state that the model is not Newtonian but rather "Newtonian-like", resulting from truncation of the fully post-Newtonian (DSX) framework. In the present paper, we deal with the Newtonian-like librations (classical physical librations), while the formally relativistic contributions (relativistic librations and de Sitter precession of the Mercury's reference frame) will be analyzed in a forthcoming paper.

\subsection{Method}

The model is solved by modular numerical integration and controlled in function of the different physical contributions and parameters taken into account. The $N$-body problem (for the translational motions), the rotational motions, the figure-figure and tidal interactions between the required bodies are simultaneously integrated with the choice of the contributions and truncations at our disposal. For instance, the upper limits of the extended figure expansions and mutual interactions may be chosen as follows: up to $l=5$ in the Moon case, 4 for the Earth, 2 for the Sun while only the Earth-Moon quadrupoleoctupole interaction is taken into account (see previous papers). The model has been especially built to favor a systematic analysis of all the effects and contributions. In particular, it permits the separation of various families of librations in the rotational motions of the bodies.

The non-linearity features of the differential equations, the degree of correlation of the studied effect with respect to its neighbors (in the Fourier space) and the spin-orbit resonances 
Table 1. Our initial conditions at 07.01.1969 (equinox J2000).

\begin{tabular}{|c|c|c|c|c|c|c|c|c|}
\hline \multicolumn{9}{|c|}{ Mercury } \\
\hline \multicolumn{9}{|c|}{ Rotation angles } \\
\hline$\psi$ & $=$ & $48.386 \mathrm{deg}$ & $\dot{\psi}$ & $=$ & $0.0 \mathrm{deg} / \mathrm{day}$ & $<\dot{\psi}>$ & $=$ & $-0.34010^{-5} \mathrm{deg} /$ day $(a)$ \\
\hline$\theta$ & $=$ & $7.005 \mathrm{deg}$ & $\dot{\theta}$ & $=$ & $0.0 \mathrm{deg} /$ day & $<\dot{\theta}>$ & $=$ & $-0.23510^{-7} \mathrm{deg} /$ day $(a)$ \\
\hline$\varphi$ & $=$ & $299.070 \mathrm{deg}$ & $\dot{\varphi}$ & $=$ & $6.138505 \mathrm{deg} /$ day $(b)$ & $\eta_{0}$ & $=$ & $1.6 \operatorname{amin}(a)$ \\
\hline
\end{tabular}

(a) Mean values derived from the SONYR model; (b) Seidelmann et al. (2002).

(in the Moon and Mercury's cases), make it hardly possible to speak about "pure" effects with their proper behavior (even after fitting of the initial conditions). The effects are not absolutely de-correlated but relatively isolated. However, the used technique (modular and controlled numerical integration, differentiation method, mean least-squares and frequency analysis) gives the right qualitative behavior of an effect and a good quantification for this effect relative to its neighbors. In the case of the particular status of the purely relativistic effetcs, their quantitative behaviors are beyond the scope of the present paper and will be discussed in a forthcoming work. When a rotational effect is simply periodic, a fit of the initial conditions for a set of given parameters only refines without really changing the effect's behavior. The amplitudes of librations plotted in Figs. 11 and 12 are then slightly upper bounds.

The precision of the model is related to the one required by the theory of the Moon. One of the aims in building the BJV model (at present included inside SONYR) was to take into account all phenomena up to the precision level resulting from the LLR data (i.e. at least $1 \mathrm{~cm}$ for the Earth-Moon distance, 1 milliarcsec (mas) for the librations). For reasons of consistency, several phenomena capable of producing effects of at least 0.1 mas had been also modeled (the resulting libration may be at the observational accuracy level). Moreover, in order to justify consistence of the Moon's theory, this one had been adjusted to the JPL ephemeris on the first $1.5 \mathrm{yrs}$ up to a level of a few centimeter residuals. In the other hand, the internal precision of the model is only limited by the numerical accuracy of the integration. Thus, in order to avoid numerical divergence at the level of our tests for Mercury, computations have been performed in quadrupole precision (32 significant figures, integration at a $10^{-14}$ internal tolerance).

\subsection{Terminology}

In order to de-correlate the different librations of Mercury, we use the terminology proposed in Bois (1995), which is suitable for a general and comparative classification of rotational motions of the celestial solid bodies. This terminology derives from a necessary re-arrangement of the lunar libration families due to both progress in the Moon's motion observations (LLR) and modern knowledge of dynamical systems.

Traditionally, the libration mode called physical libration is split up according to the conventional dualism "forced-free". The forced physical librations are generally related to gravitational causes while the free librations would be departures of the angular position from an equilibrium state. These cuttings out contain ambiguities and redundancies discussed in previous papers (Bois 1995, 2000). Formally, the free librations are periodic solutions of a dynamical system artificially integrable (by a convention of writing related to specific rates of the spinorbit resonance, for instance 1:1), whereas the forced librations express, in space phase, quasi-periodic solutions around a fixed point (the system is no longer integrable). Moreover, any stable perturbed rotation of celestial solid body contains imbricate librations of different nature, and those are too strongly overlapped to keep the traditional classification.

In the present terminology, the libration nature, its cause and its designation are linked up. Two great libration families serve to define the physical librations, namely the potential librations and the kinetical librations. They simply correspond to a variation energy, potential or kinetical respectively. For libration sub-classes, the designation method is extensive to any identified mechanism (see more details in Bois 1995). The terminology permits easily the separation of various families (see the Moon's case described in a set of previous papers). These librations are called direct when they are produced by torques acting on the body's rotation. They are called indirect when they are produced by perturbations acting on the orbital motion of the body. Indirect librations derive from spin-orbit couplings.

A specificity of the SONYR model with its method of analysis is to isolate the signature of a given perturbation. The SONYR model allows indeed the identification of relationships between causes and effects including interactions between physics and dynamics, such as the dynamical signature of a core-mantle interaction (called centrifugal librations).

\subsection{Parameters and initial conditions}

In the computations presented in the paper, the required dynamical parameters and general initial conditions come from the JPL DE405 ephemeris (Standish 1998). However, concerning the parameters related to the Mercury's rotation (seconddegree spherical harmonics $C_{20}$ and $C_{22}$ ), which are not included in the JPL ephemeris, our model uses those given by Anderson et al. (1987) (see Table 2). Besides, up to now it does not exist any ephemeris of the Mercury's rotation. As a consequence, to build initial conditions for the Hermean rotation (described by an Eulerian sequence of angles $\psi, \theta$ and $\varphi$ defined below in Sect. 4.1), we use the following principle: assuming the polar axis of Mercury normal to its orbital plane, we obtain $\psi=\Omega$ and $\theta=i$ where $\Omega$ and $i$ are respectively the ascending node and the inclination of the orbit of Mercury on the Earth 
Table 2. Parameters of Mercury.

\begin{tabular}{lrr}
\hline \hline \multicolumn{3}{c}{ Mercury } \\
Bulk quantities \\
\hline Mass $\left(G M_{\odot}\right)$ & $=$ & $4.9125 \times 10^{-10}(a)$ \\
Equatorial radius $(\mathrm{km})$ & $=$ & $2439(b)$ \\
$J_{2}$ & $=$ & $(6.0 \pm 2.0) \times 10^{-5}(b)$ \\
$C_{22}$ & $=$ & $(1.0 \pm 0.5) \times 10^{-5}(b)$ \\
$C / M R^{2}$ & $=$ & $0.34(c)$ \\
\hline
\end{tabular}

(a) JPL; (b) Anderson et al. (1987); (c) Milani et al. 2001.

Table 3. Our results for the spin-orbit motion of Mercury. The spinorbit period verifies the relation: $\widetilde{P}=2 P_{\lambda}=3 P_{\varphi}$.

\begin{tabular}{llr}
\hline \hline \multicolumn{3}{c}{ Mercury } \\
\multicolumn{3}{c}{ Spin-orbit characteristic period } \\
\hline$\Phi(1$ st proper frequency) & $=$ & $15.847 \mathrm{yrs}$ \\
$\Psi$ (2nd proper frequency) & $=$ & $1066 \mathrm{yrs}$ \\
$\Pi$ (orbital precession) & $=$ & $278898 \mathrm{yrs}$ \\
$P_{\lambda}$ (orbital period) & $=$ & 87.969 days \\
$P_{\varphi}$ (rotational period) & $=$ & 58.646 days \\
$\widetilde{P}($ spin-orbit period $)$ & $=$ & 175.95 days \\
\hline
\end{tabular}

equatorial plane (which is the reference frame used in the DE405 ephemeris). The long axis of Mercury being pointed towards the Sun at its periapse allows to fix the $\varphi$ angle of polar rotation. The value of $\dot{\varphi}$ is found in Seidelmann et al. (2002). We use at last $\dot{\psi}=0$ and $\dot{\theta}=0$; these two variables reach to mean values generated by the complete spin-orbit problem of Mercury: $-0.340 \times 10^{-5} \mathrm{deg} / \mathrm{day}$ and $-0.235 \times 10^{-7} \mathrm{deg} / \mathrm{day}$ respectively. The numerical integrations presented in the paper start from these initial conditions related to the planar problem for Mercury; they are listed in Table 1. Departure from the planar case is understood as the integration of physics included in SONYR.

In the other hand, for the computations carried out in this paper, the global reference frame $O^{\prime} X^{\prime} Y^{\prime} Z^{\prime}$ is given by a reference system centered on the solar system barycenter, fixed on the ecliptic plane, and oriented at the equinox $J 2000$. The rotational motion of Mercury is evaluated from a coordinate axis system centered on the Mercury's center of mass $O x y z$ relative to a local dynamically non-rotating reference frame, $O X Y Z$, whose axes are initially co-linear to those of $O^{\prime} X^{\prime} Y^{\prime} Z^{\prime}$. In the framework of the present paper without purely relativistic contributions, let us note that axes of $O X Y Z$ remain parallel to those of $O^{\prime} X^{\prime} Y^{\prime} Z^{\prime}$.

The $N$-body problem for the planets of the solar system and the Mercury's spin-orbit motion are simultaneously integrated in the SONYR model. Concerning the rotational equations written in a relativistic framework, the reader may refer to Bois \& Vokrouhlický (1995). In a Newtonian approach, these equations amount to the classical Euler-Liouville equations of the solid rotation (see e.g. Goldstein 1981). We follow the formalism and the axiomatic expanded in Bois \& Journet (1993) and Bois (1995) for the definition of the different rotational elements as well as the used terminology. Let us simply precise that $\mathbf{l}$ is the angular momentum expressed in $O x y z$ and is related to the instantaneous rotation vector $\omega$ as follows:

$\mathbf{l}=(I) \omega$

where $(I)$ is the tensor of inertia for the body. According to classical assumptions, $(I)$ is reduced to three principal moment of inertia $A, B$, and $C$. The gravity field of Mercury is essentially unknown. The tracking data from the three fly-by of Mariner 10 in 1974-75 have been re-analyzed by Anderson et al. (1987) to give a low accuracy estimate of the normalized coefficients $C_{20}$ and $C_{22}$ (see Table 2, values are expressed in the body-fixed frame of the principal axes of inertia). The principal moments of inertia $A$ and $B$ are then infered from $C_{20}$ and $C_{22}$ by the following formulae (Ferrari et al. 1980):

$$
\begin{gathered}
\frac{A}{M R^{2}}=C_{20}-2 C_{22}+\frac{C}{M R^{2}} \\
\frac{B}{M R^{2}}=C_{20}+2 C_{22}+\frac{C}{M R^{2}} .
\end{gathered}
$$

Let us note that the parameter $C / M R^{2}$ is not constrained within these relations. We present in Sect. 4.2 the variations of $C / M R^{2}$ by analyzing its signature into the librations of Mercury.

\section{The librations of Mercury}

\subsection{Planetary perturbations}

For Mercury assumed to be a rigid body reduced to three oblateness coefficients, the general expression for a torque coming from a disturbing point mass $m$ at the vectorial position $r \boldsymbol{u}$ ( $r$ is the instantaneous distance) is written as follow:

$\boldsymbol{N}_{2}=\left(\frac{3 G m}{a^{3}}\right)\left(\frac{a}{r}\right)^{3} \boldsymbol{u} \times(I) \boldsymbol{u}$

where $a$ is the mean distance between the two bodies. Such a torque due to the Sun is the dominant one acting on the rotational motion of Mercury. The solar torque is indeed responsible of the global dynamical behavior of the rotation of Mercury. Due to Venus, the resulting torque is about of the order of $10^{5}$ times smaller, that is quite negligible.

Figure 4 presents the rotational motion of Mercury including only the solar torque in the rotational equations and taking into account simultaneously the whole $N$-body problem for the Sun and the planets (the planetary interactions inducing indirect effects on the rotation of Mercury). In this figure, the Euler angles $\psi, \theta, \varphi$ related to the 3-1-3 angular sequence describe the evolution of the body-fixed axes $O x y z$ with respect to the axes of the local reference frame $O X Y Z$. Let us recall the definition used for these angles: $\psi$ is the precession angle of the polar axis $O z$ around the reference axis $O Z, \theta$ is the nutation angle representing the inclination of $O z$ with respect to $O Z$, and $\varphi$ is the rotation around $O z$ and conventionally understood as the rotation of the greatest energy (it is generally called the proper rotation). The axis of inertia around which is applied the proper rotation is called the axis of figure and defines the North pole of the rotation (Bois 1992). Let us remark that in Fig. 4 (in other figures involving $\varphi$ as well) plotted over $3000 \mathrm{yrs}$, 

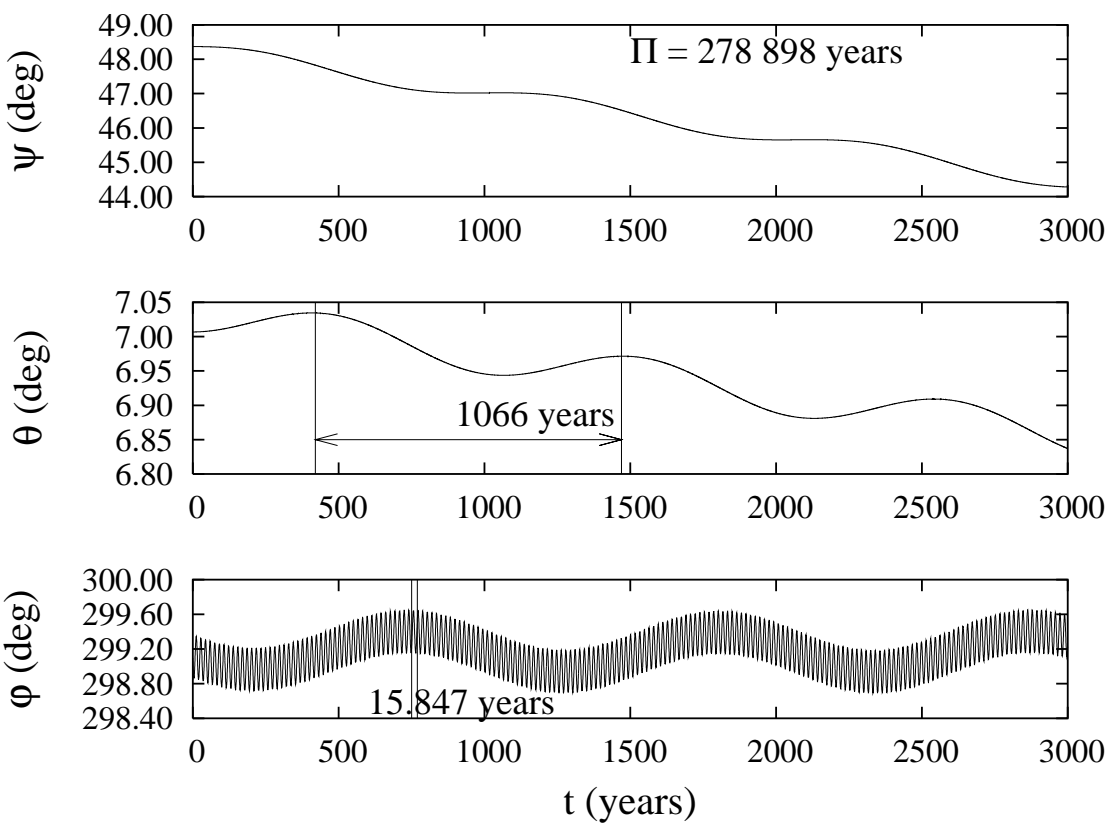

Fig. 4. The rotational motion of Mercury expressed in the ecliptic reference frame $O X Y Z$ by using the 3-1-3 Eulerian sequence $(\psi, \theta, \varphi)$, and plotted over 3000 yrs. Degrees are on the vertical axis and years on the horizontal axis. The value of 278898 yrs for the secular part of the $\psi$ angle is related to the planetary interactions. The period of 1066 yrs in the three angles is the libration of the spin-orbit secular variable. The period of $15.847 \mathrm{yrs}$ in the $\varphi$ angle is the proper frequency in longitude of Mercury.

we have removed the mean rotation of 58.646 days in the $\varphi$ angle in order to better distinguish the librations. We may then clearly identify the first proper frequency of $15.847 \mathrm{yrs}$ (to be compared to the analytical determination, namely $15.830 \mathrm{yrs}$ given in Sect. 2).

The $\psi$ angle expresses the nodal precession of the equatorial plane of Mercury with respect to the ecliptic plane. It splits up in a periodic term with a period $\Psi=1066 \mathrm{yrs}$ and a secular one $\Pi=278898 \mathrm{yrs}$. $\Psi$ is the second proper frequency of the Mercury's spin-orbit coupling. It can be analytically approximated by the following formula (used in the Earth's case by Goldstein 1981):

$2 \pi /\left(\frac{3}{2} \frac{n^{2}}{\omega_{z}} \frac{C-\bar{A}}{C} \cos \theta\right)$

This analytical expression is suitable for an axis symmetric body. It is not the case of Mercury. However, assuming Mercury as a symmetric top rotating about its smallest axis of inertia, with an average equatorial moment of inertia $\bar{A}=\frac{A+B}{2}$, one finds for this period $1300 \mathrm{yrs}$ (by using the values given in Table 2). The difference between the two values permits to appreciate the departure of Mercury from a symmetric body. In the other hand, the dynamical behavior of $\psi$ coming from SONYR is due to the direct effect of the solar torque by the way of the true dynamical figure of Mercury. In the 2-body problem, Sun-Mercury, $\Psi=1066$ yrs and $\Pi=0$ yrs. Related to the planetary interactions, $\Pi=278898$ yrs expresses the departure from the 2-body problem.

The dynamical behaviors of $\psi$ and $\Omega$ (the ascending node of the orbit) are quite superimposed as shown in Fig. 5 (top panel). As it is mentioned by Béletski (1986), a second synchronism is generally involved in a Cassini state. However, in the Mercury's
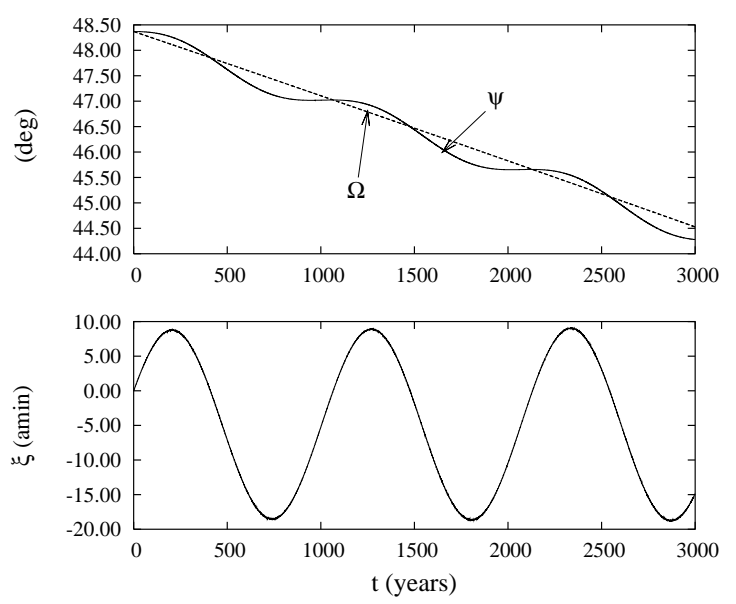

Fig. 5. Mercury's spin-orbit secular resonance. The top panel presents the evolution of $\psi$ and $\Omega$ both including a secular term. The bottom panel presents the behavior of $\xi=\psi-\Omega$ plotted over $3000 \mathrm{yrs}$. Let us underline that this $\xi$ angle does not present any secular term, showing then a synchronism between both angles, $\psi$ and $\Omega$. On the top pannel, degrees are on the vertical axis while arcminutes are on the bottom panel; years are on both horizontal axes.

case, it is not a periodic synchronism as it is the case for the Moon. Using SONYR, we make easily in evidence the periodic $18.6 \mathrm{yr}$ synchronism in the lunar spin-orbit resonance while it is about a secular behavior in the Hermean spin-orbit resonance. The 3:2 resonance of Mercury introduces a mechanism of spin-orbit secular resonance qualitatively analogous to the orbital secular resonances combined with mean motion resonances (as for instance in the 2:1 case, see Bois et al. 2003). As it is shown in Fig. 5 (bottom panel), the spin-orbit secular resonance variable $\xi=\psi-\Omega$ does not present any secular term. As a 

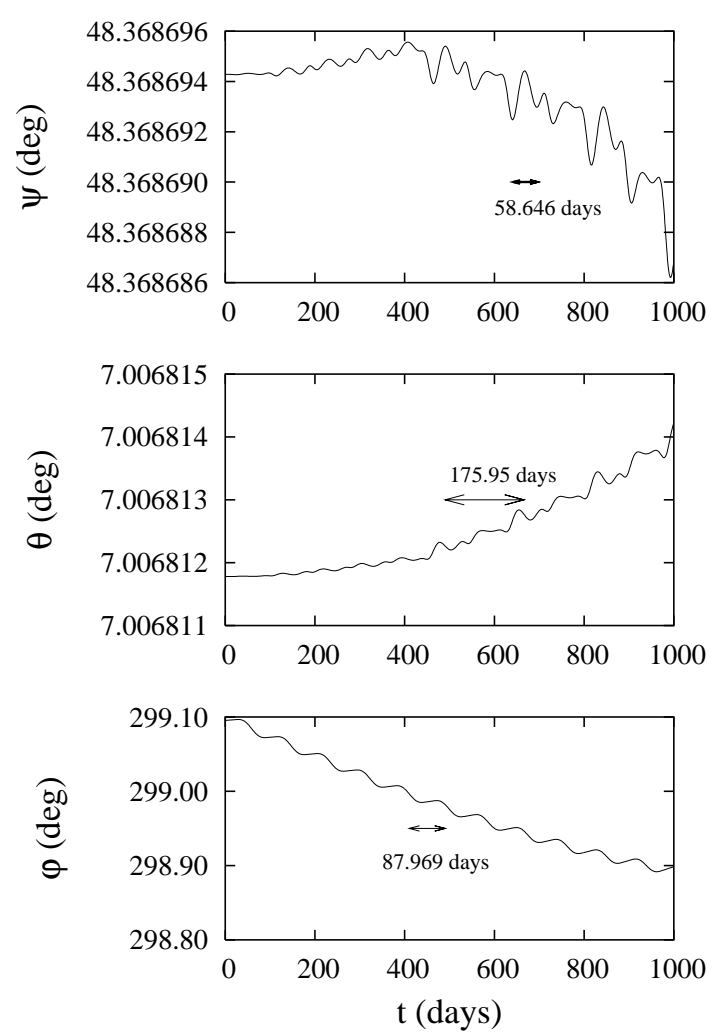

Fig. 6. The rotational motion of Mercury expressed in the ecliptic reference frame $O X Y Z$ by using the 3-1-3 Eulerian sequence $(\psi, \theta, \varphi)$, and plotted over 1000 days. Degrees are on the vertical axes and days on the horizontal axis. The periods of 58.646 and 87.969 days in the $\psi$ and $\varphi$ angles respectively, express precisely the 3:2 spin-orbit resonance rate. The period $\tilde{P}=175.95$ days appears clearly in these plots.

consequence, $\psi$ and $\Omega$ on average precess at the same rate equal to $\Pi$, confirming then the mechanism of spin-orbit secular resonance. We find that $\xi$ librates with the particular frequency of 1066 yrs.

In addition, in order to give a detailed inspection of the short periods involved in the rotational motion of Mercury, Fig. 6 presents the solution plotted over 1000 days. The $P_{\varphi}$ rotation period of 58.646 days appears in the $\psi$ and $\theta$ angles. Whereas the mean rotation of 58.646 days is removed in the $\varphi$ angle (as in Fig. 4), the signature of the $P_{\lambda}$ orbital period of 87.969 days is clearly visible (this angle is called libration in longitude of 88 days in literature). A third period appears in the $\psi$ and $\theta$ angles, namely 175.95 days. This one results from the 3:2 spin-orbit resonance $\left(\widetilde{P}=2 P_{\lambda}=3 P_{\varphi}\right)$.

Figure 7 presents the planetary interactions acting on the rotational motion of Mercury by the way of its spin-orbit couplings (i.e. indirect effects of the planets on the Mercury's rotation). In the black line case the problem is reduced to the Sun and Mercury. In this 2-body problem, the orbital plane does not precess as it is clear in the $\psi$ and $\theta$ angles without secular terms. The secular variations rise up from the departure of the 2-body problem (as it is visible with the broken, dots, and cross line cases in Fig. 7). In the broken line case, the interactions between the Sun, Mercury and Venus are taken into account. With the dots line, the later case includes Jupiter in addition. The whole planetary interactions are integrated in the cross line case (except for Pluto). We show that Venus is the planet which induces the greatest secular term. After Venus, the role of Jupiter is dominant, and this 4-body problem (Sun, Mercury, Venus, and Jupiter) defines our "standard" case used in our Sect. 4.3 for the analysis of the Hermean librations. The rate of secular variations in the Mercury's rotation between all planetary interactions (cross lines) and our standard case (dots lines) is 11.8 as/cy (as: arcseconds) in the $\theta$ nutation angle and $1.9 \mathrm{amin} / \mathrm{cy}$ (amin: arcminutes) in the $\psi$ precession angle. These values should be used as corrective terms in analytical theories of the rotational motion of Mercury. Let us emphasize that the spin-orbit motion of Mercury coming from our standard case is sufficient for preserving the 3:2 resonance ratio between the two modes of motion.

Starting with the initial conditions defined in Sect. 3.4 (where in particular the initial obliquity of Mercury is equal to zero), the SONYR model permits obtaining the dynamical behavior of the Hermean obliquity by its simultaneous spin-orbit integration. The variables $i, \theta, \Omega, \psi$ from SONYR substituted in the following relation:

$\cos \eta=\cos i \cos \theta+\sin i \sin \theta \cos (\Omega-\psi)$

produce the instantaneous obliquity $\eta$ plotted over $3000 \mathrm{yrs}$ as presented in Fig. 8. Such a behavior for $\eta$ gives easily a mean obliquity $\eta_{0}=1.6$ amin. We show at present that this mean value is quite consistent with the Cassini state of Mercury. Let be the following equation established by Peale (1988) and reformulated by Wu et al. (1997) and coming from the Cassini laws:

$\frac{M R^{2}}{C}=\frac{\mu}{n} \frac{\sin \left(i+\eta_{0}\right)}{\sin \eta_{0}\left[\left(1+\cos \eta_{0}\right) G_{201} C_{22}-\cos \eta_{0} G_{210} C_{20}\right]}$

where $\mu$ is the precessional angular velocity of the Hermean orbit, namely $2 \pi / \Pi \mathrm{yrs}^{-1}$, while

$G_{201}=\frac{7}{2} e-\frac{123}{16} e^{3}$ and $G_{210}=\left(1-e^{2}\right)^{-\frac{3}{2}}$

are eccentricity functions defined by Kaula (1966). $i$ is the inclination of the orbital plane of Mercury relative to a reference system precessing with the orbit. This inclination varies between from $5^{\circ}$ to $10^{\circ}$ while the eccentricity varies from 0.11 to 0.24 , over $10^{6}$ yrs (Peale 1988). As a consequence, $\eta_{0}$ obtained with (14) belongs to [1.33, 2.65] amin. Let us note that a conventional value of 7 amin is often given in literature. Such a value, outside the interval of possible values, is very probably incorrect as already claimed by Wu et al. (1997).

\subsection{Principal figure librations}

\subsubsection{Signature of the $C / M R^{2}$ coefficient on the rotational motion of Mercury}

Let us consider at present the disturbing torques acting on the rotational motion of Mercury and as a consequence inducing direct librations. This section focuses on the librations related to the dynamical figure of the planet. Such librations are called principal figure librations (Bois 1995). We assume the Sun reduced to a point mass while the gravity field of Mercury is expanded in spherical harmonics up to the degree 2 . We express 

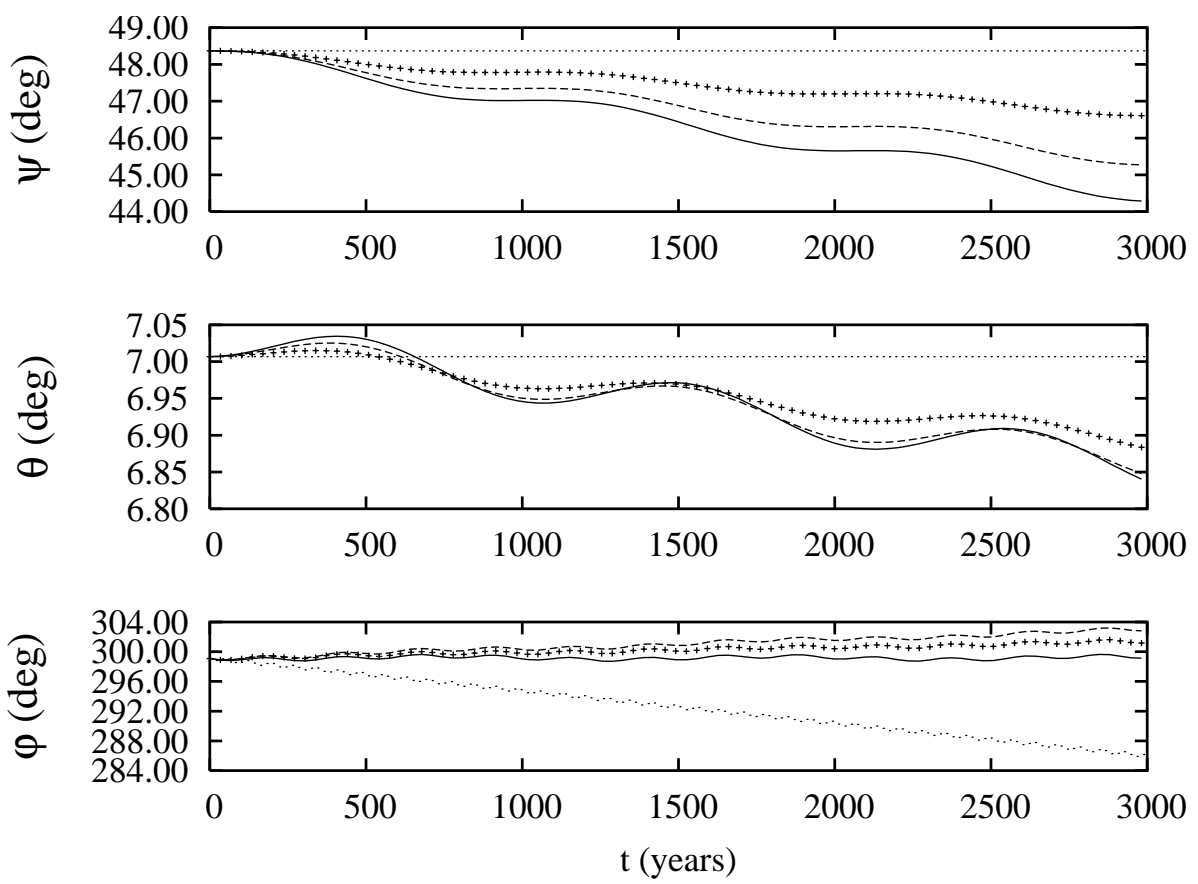

Fig. 7. Interactions of the planets on the Hermean rotational motion by the way of the spin-orbit couplings over 3000 yrs. Degrees are on the vertical axes and years on the horizontal axis for the three panels. In the black line case, the problem is reduced to the Sun and Mercury. In the broken line case, the interactions between the Sun, Mercury and Venus are taken into account. With the dots line the later case includes Jupiter in addition. The whole planetary interactions are integrated in the cross line case (except for Pluto). The dots line case defines our called standard case sufficient for preserving the 3:2 resonance ratio.

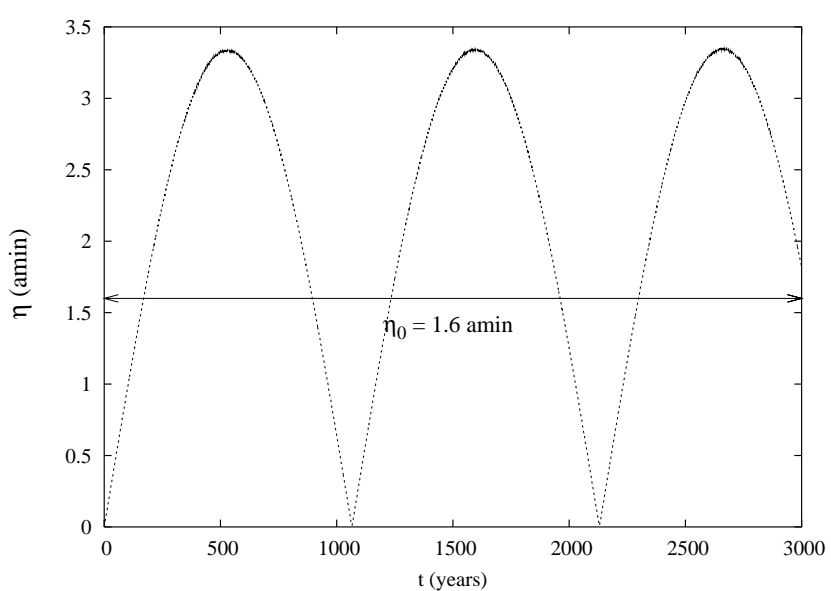

Fig. 8. Dynamical behavior of the $\eta$ instantaneous obliquity of Mercury plotted over 3000 yrs. Arcminutes are on the vertical axis and years on the horizontal axis. Over this time of integration, the behavior is simply described by a period of $1066 \mathrm{yrs}$ and 3.2 amin of amplitude. The $\eta_{0}$ mean obliquity is 1.6 amin.

the solar torque acting on the figure of Mercury according to Eq. (11).

The first coefficients of the Hermean gravity field have been determined with the Mariner 10 probe (Anderson et al. 1987). We use these values for $C_{20}=-J_{2}$ and $C_{22}$ given in Table 2. In order to complete the Hermean tensor of inertia (coefficients $A, B, C$ ), the $C / M R^{2}$ principal moment of inertia is required (see Eq. (11)). Its value is related to the internal density distribution of the planet according to the polar axis of Mercury (rotation of greatest energy about the smallest principal axis of inertia). For an homogeneous planet, such a normalized value is equal to 0.4 . We use a nominal value of 0.34 (Table 2) used by Milani et al. (2001) and coming from an internal structure model of Mercury including three layers (crust, mantle and core).

Figure 9 presents the rotational behavior of Mercury computed over 10000 yrs in our standard case with two different values of its greatest principal moment of inertia: (i) the computation with $C / M R^{2}=0.4$ is plotted with the dashed lines (homogeneous planet); (ii) the broad lines are obtained with $C / M R^{2}=0.34$ (three layer model of Mercury). On the bottom panel ( $\varphi$ angle), the dashed lines are shifted away 1 degree in order to distinguish them from the broad lines. Figure 9 (especially the $\theta$ angle) shows how the $C / M R^{2}$ coefficient value and the constant of precession $\Psi$ (2nd proper frequency) are related. With $C / M R^{2}=0.4, \Psi=1254.01$ yrs while with $C / M R^{2}=0.34$, $\Psi=1066$ yrs. Besides, $C / M R^{2}$ and $\Phi$ (1st proper frequency) are also linked and for evaluating this relation, Fig. 10 shows the variations $\Delta \varphi$ obtained by differentiation: (i) on the top panel $\Delta C=1 \%$, (ii) on the middle panel $\Delta C=2 \%$, (iii) on the bottom panel $\Delta C=15 \%(0.34+15 \%(0.34)=0.4)$. The beats are signatures related to the variations in $\Phi$.

\subsubsection{Signature of the $C / M R^{2}$ coefficient on the obliquity and on the libration angle in longitude}

One of the main objectives of the BepiColombo and MESSENGER missions is to measure the rotation state of Mercury, up to an accuracy allowing to constrain the size and 

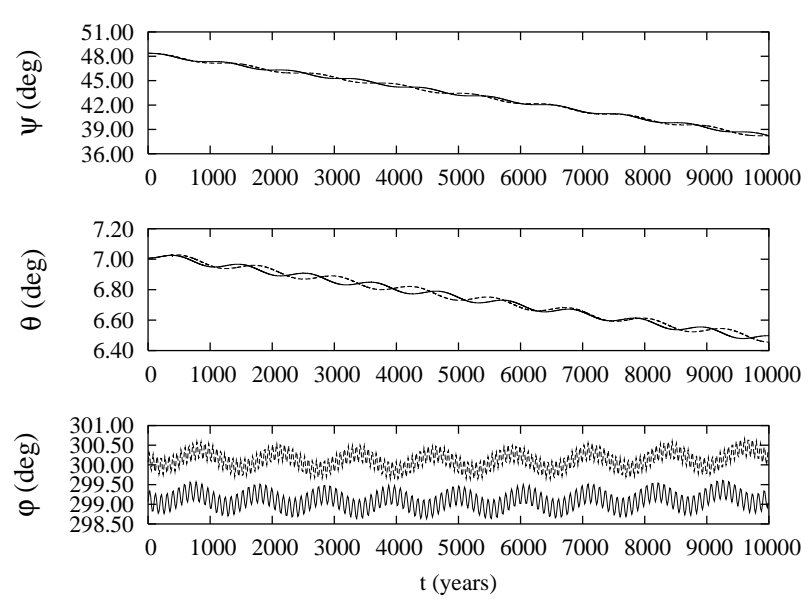

Fig. 9. Rotational behavior of Mercury for two different values of its greatest principal moment of inertia. Degrees are on the vertical axes and years on the horizontal axes. The computation with $C / M R^{2}=0.4$ is plotted through the dashed lines; this value corresponds to an homogeneous planet. The broad lines are obtained with $C / M R^{2}=0.34$; this value derives from an internal structure model of Mercury including three layers. On the bottom panel ( $\varphi$ angle), the dashed lines are shifted away 1 degree in order to distinguish them from the broad lines.

physical state of the planet's core (Milani et al. 2001; Solomon et al. 2001). Consequently, the two missions have to determine the four following parameters: $C_{20}, C_{22}, \eta$, and $\varphi$ that are sufficient to determine the size and state of the Mercury's core (see Peale 1988, 1997). Combining $C_{20}, C_{22}$, and $\eta$, one obtains the $C / M R^{2}$ coefficient while with $C_{22}$ and $\varphi$, one obtains $C_{m} / M R^{2}$ (i.e. the $C / M R^{2}$ coefficient for the mantle). The validity condition of the first combination is that the dynamical behavior of the core has to follow the one of the mantle over a period of time at least the one of $\Pi$ (assertion 1 ). The validity condition of the second combination is that the dynamical behavior of the core has not to be coupled to the one of the mantle over a period of 88 days $\left(P_{\lambda}\right)$ (assertion 2$)$. These two conditions linked together imply some constraints on the nature of the core-mantle interface (Peale 1997). In order to reach such an objective, the BepiColombo mission has to obtain a value on the $C / M R^{2}$ coefficient with an accuracy of 0.003 , i.e. $1 \%$ and therefore foresees measuring the libration angle and the obliquity with an accuracy of 3.2 and 3.7 as respectively (Milani et al. 2001).

Our SONYR model gives (i) the true relation between the three parameters $\left(C / M R^{2}, \eta, \varphi\right)$, and (ii) the upper bounds of the impact of $C_{m} / M R^{2}$ on the $\varphi$ angle. Figure 11 presents the impact of the $C / M R^{2}$ coefficient on the instantaneous obliquity $\eta$. In these plots (Figs. 11 and 12), the spin-orbit motion of Mercury is integrated within the whole solar system with an initial obliquity of 1.6 amin, which is the mean obliquity of Mercury evaluated in Sect. 4.1. The top panel of Fig. 11 expresses the dynamical evolution of $\eta$ computed over 500 days with $C / M R^{2}=0.34$ (black lines) and $C / M R^{2}=0.3434$ (dashed lines). Dashed lines are shifted from 0.001 amin in order to distinguish the two different kinds of lines. Figure 11 shows also how the instantaneous obliquity of Mercury differs from its 1.6 amin nominal value. The bottom panel shows by
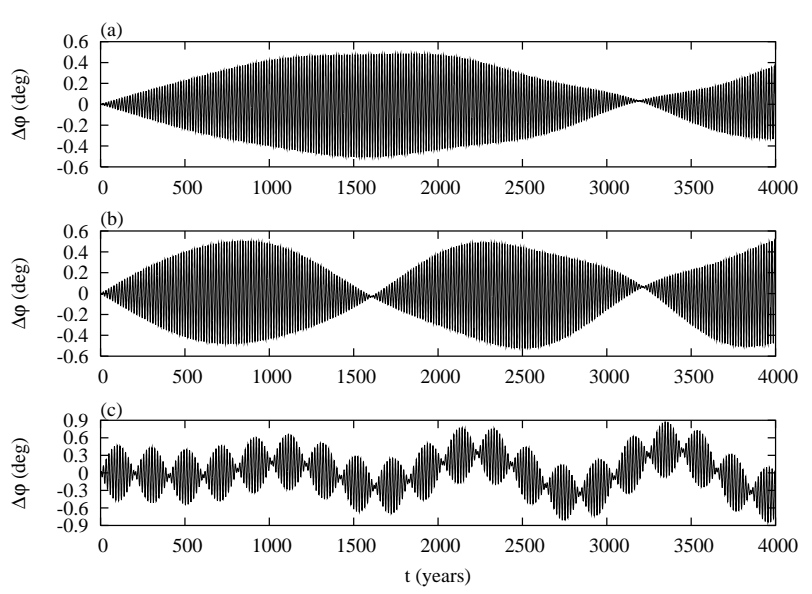

Fig. 10. The signature of the libration of Mercury when the principal moment of inertia along the axis of figure varies from $1 \%$ in the top panel a), to $2 \%$ in the mean panel and $15 \%$ in the bottom panel c). Degrees are on the vertical axes and years on the horizontal axes.

differentiation the signature of the $1 \%$ variation of $C / M R^{2}$ on $\eta$. The maximal amplitude crest to crest is of the order of 2.3 mas within the characteristic period of 175.95 days related to the 3:2 ratio of the Mercury's spin-orbit resonance.

Figure 12 presents the signature of the $C / M R^{2}$ coefficient on the $\varphi$ libration angle in longitude. The top panel expresses the behavior of $\varphi$ computed over 500 days with $C / M R^{2}=0.34$ (black lines) and $C / M R^{2}=0.3434$ (dashed lines). One may compare this Fig. 12 to Fig. 3 resulting from the usual analytical resolution of the Eulerian Eq. (4) (thanks to G. Giampieri, private communication). Let us note that the angle $\gamma$ defined in Fig. 3 is equal to the angle $\varphi$ plotted in Fig. 12. The later only gives a simple double sine curve with an amplitude of 42 as while the solution of the SONYR model includes the couplings between the three rotational variables as well as the indirect couplings due to planetary interactions (we notice that in the two Figs. 12 and 3 the amplitude of libration is of the order of 40 as). Let us note that Fig. 12 corresponds to the libration related to the $C / M R^{2}$ coefficient of the planet without core-mantle couplings. Let us add that in Peale (1972), the amplitude of $\varphi$ is related to the $C_{m}$ coefficient by assuming that the assertion 2 quoted upper is true. On the contrary, our first results on this topic make in evidence the existence of a faint coupling. This core-mantle coupling will be presented in a forthcoming paper. The bottom panel of Fig. 12 shows by differentiation the signature of the $1 \%$ variation of $C / M R^{2}$ on $\varphi$. The maximal amplitude within the period of about 88 days (i.e. the signature of $P_{\lambda}$ ) is of the order of 0.45 as.

In conclusion, signatures of the indeterminacy of $1 \%$ in $C / M R^{2}$ on the obliquity and on the libration in longitude are 2.3 mas and 0.45 as respectively. What is very faint (may be too much) with respect to the expected accuracy forecasted in the BepiColombo mission.

\subsection{Varying the obliquity}

Because the initial obliquity value is unknown, we test in this last section the impact of the indeterminacy of this value on 

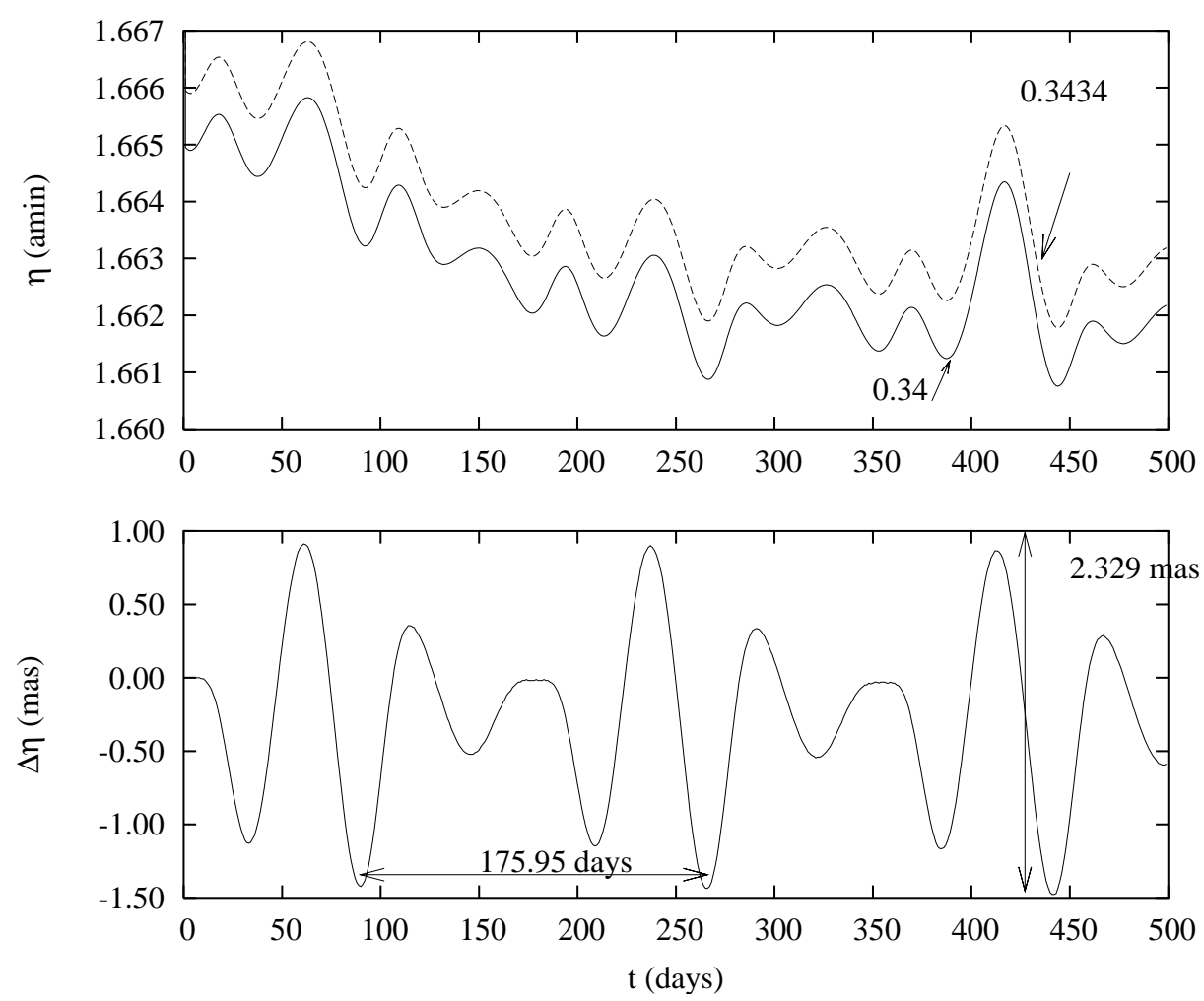

Fig. 11. Signature of the $C / M R^{2}$ coefficient on the obliquity of Mercury for two different values shifted from $1 \%$. Arcminutes are on the vertical axis of the top panel while milliarcseconds are on the vertical axis of the bottom panel; days are on the horizontal axis for both panels. The maximal amplitude of the signature of $C / M R^{2}$ on the obliquity is 2.3 mas. On the top panel, the dashed lines are shifted away 0.001 amin in order to distinguish them from the broad lines.
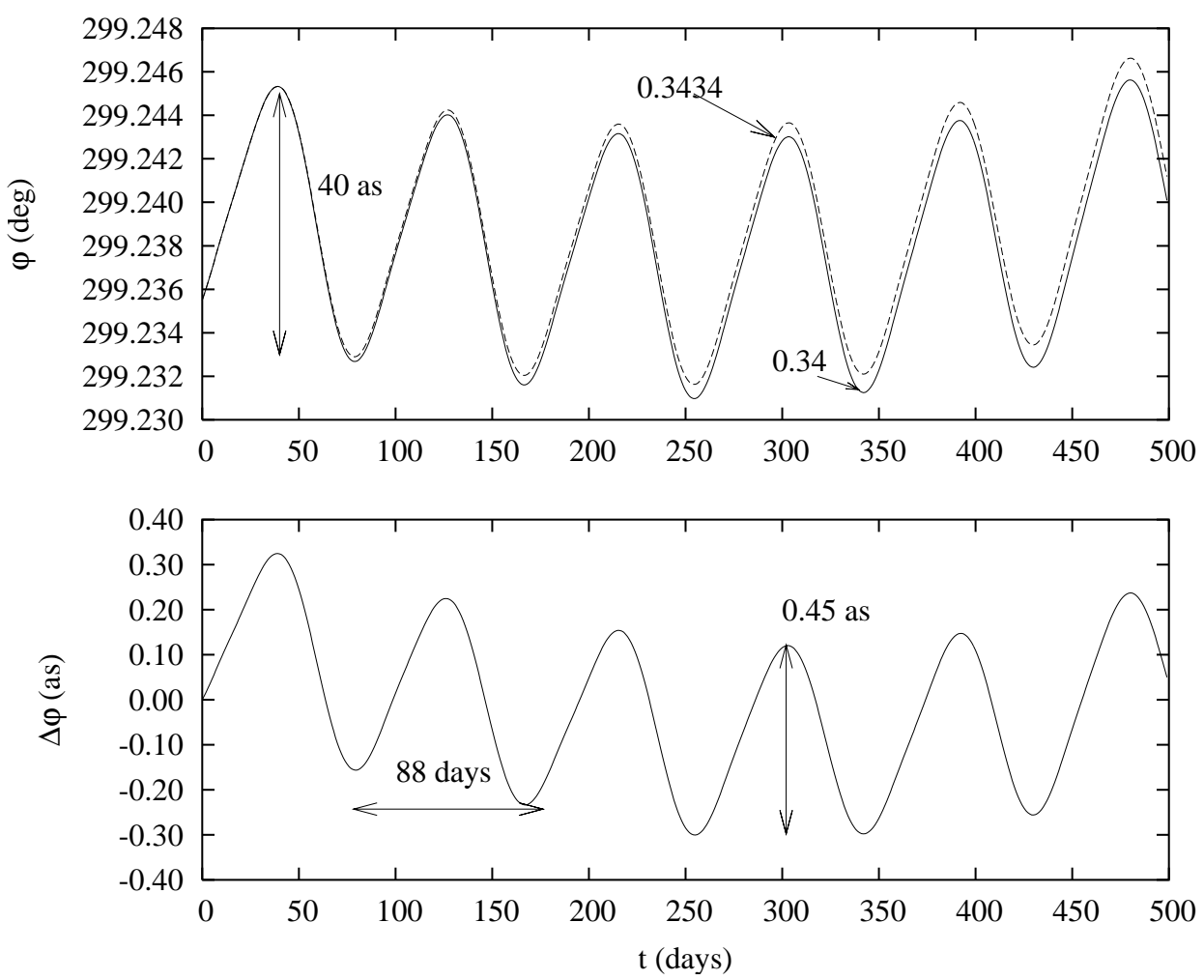

Fig. 12. Signature of the $C / M R^{2}$ coefficient on the Hermean libration angle in longitude for two different values shifted from $1 \%$. Degrees are on the vertical axis of the top panel and arcseconds are on the vertical axis of the bottom panel; days are on the horizontal axis for both panels. The maximal amplitude of the signature of $C / M R^{2}$ on the libration angle in longitude is 0.45 as. 

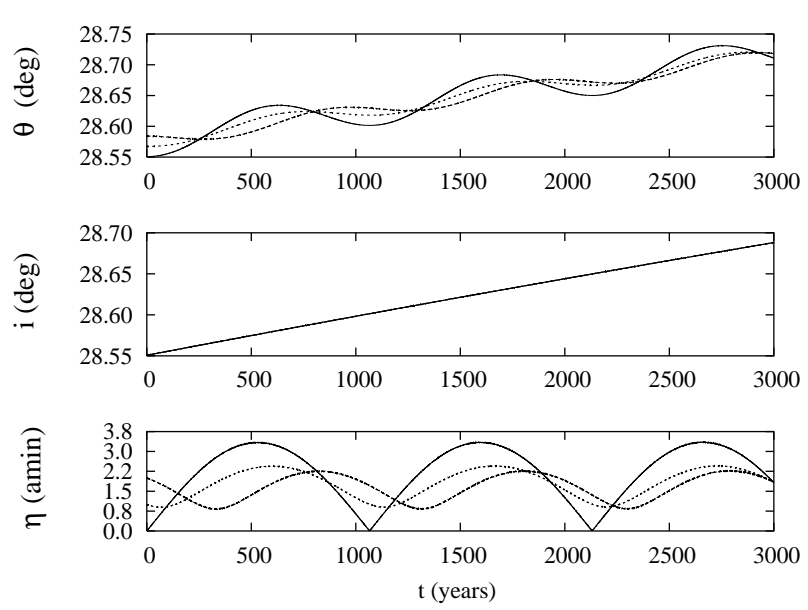

Fig. 13. Impact of the initial obliquity on the nutation angle $\theta$ (top panel), on the orbital inclination $i$ (middle panel), and on the instantaneous obliquity $\eta$ (bottom panel). Degrees are on the vertical axes of the top and middle panels while arcminutes are on the vertical axis of the bottom panel; years are on all horizontal axes. On each panel, three curves plotted over 3000 yrs correspond to three different initial values of $\eta$ : 0 amin (black lines), 1 amin (dashed lines), and 2 amin (dot lines).

the spin-orbit motion of Mercury. The results are presented in Fig. 13 plotted over $3000 \mathrm{yrs}$; top panel: the effect on the nutation angle $\theta$, middle panel: the effect on the orbital inclination $i$, and bottom panel: the effect on the instantaneous obliquity $\eta$. On each panel, three curves are related to three different initial values of $\eta$, namely 0 amin (black lines), 1 amin (dashed lines), and 2 amin (dot lines). In the bottom panel, the amplitudes of these librations are of the order of 1.4 amin with a period of 1066 yrs. For any initial value of $\eta \in[0,3.2]$ amin, the mean value of $\eta$, let be $\eta_{0}$, is equal to 1.6 amin, which is in good agreement with the determination of $\eta_{0}$ in a previous section. We may claim that $\eta_{0}=1.6$ amin.

For obtaining such a mean obliquity by measurements, let us underline that the theoretical behavior of $\eta$ points out to fit the observations by a sine function taking into account the long period $\Psi=1066$ yrs with an amplitude of 1.6 amin.

\section{5. conclusion}

The 3:2 spin-orbit resonance between the rotational and orbital motions of Mercury results from a functional dependance of the tidal friction adding to a non-zero eccentricity and a permanent asymmetry in the equatorial plane of the planet. The upcoming space missions, MESSENGER and BepiColombo with onboard instrumentation capable of measuring the rotational parameters stimulate the objective to reach an accurate theory of the rotational motion of Mercury.

Starting from our BJV relativistic model of solar system integration including the coupled spin-orbit motion of the Moon, we have obtained a model generalizing the spin-orbit couplings to the terrestrial planets (Mercury, Venus, Earth, and Mars). The updated model is called SONYR (acronym of Spin-Orbit $N$-BodY Relativistic model). It permits to analyze and identify the different families of rotational librations. This work has been carried out for Mercury in the present paper.
The spin-orbit motion of Mercury is characterized by two proper frequencies (namely $\Phi=15.847$ and $\Psi=1066 \mathrm{yrs}$ ) and its 3:2 resonance presents a second synchronism which can be understood as a spin-orbit secular resonance, $(\Pi=$ $278898 \mathrm{yrs}$ ). A new determination of the mean obliquity has been proposed in the paper. By using the SONYR model, we have found a mean obliquity of $1.6 \mathrm{amin}$. This value is consistent with the Cassini state of Mercury. Besides, we have identified in the Hermean librations the impact of the uncertainty of the greatest principal moment of inertia $\left(C / M R^{2}\right)$ on the obliquity and on the libration in longitude (2.3 mas and 0.45 as respectively for an increase of $1 \%$ on the $C / M R^{2}$ value). These determinations prove to be suitable for providing constraints on the internal structure of Mercury. The direct core-mantle interactions will be presented in a forthcoming paper.

Acknowledgements. The authors thank A. Pavlov for his help in the Poincaré cross-section computations and J. Brillet for providing his efficient method of mean last squares useful for accurate determinations of periods in our data files.

\section{Appendix A: Free rotation}

Let us assumed Mercury isolated in space; in this sense, its rotation is free and the Euler-Liouville equations for its rotation are written without right hand side, i.e. without any external disturbing torques. If we add the assumption of a rigid Mercury, we are in the Euler-Poinsot motion case (whose solutions are the well-known Eulerian oscillations). Without explicitly integrating such equations, the assurance of integrability in the Poincare sense can be obtained by some theoretical simple considerations. Indeed, whatever being the triplet of generalized coordinates used to describe the spatial attitude of a solid body in a fixed frame, one knows that there exists four independant integrals of motion: the Hamiltonian $H$, and the three components $L_{X}, L_{Y}, L_{Z}$ of the angular momentum (in $O X Y Z$ ). Four integrals of motion for three degrees of freedom, the problem is then integrable and even over-integrable. One does not lose the generality of the problem choosing for instance $A \leq B \leq C$. The choice $A \leq B<C$ makes possible to write the general solution of the system under a form involving the elliptical functions of Jacobi (Landau \& Lifchitz 1969). By convention, let us adopt that the resulting oscillations in space be called the Eulerian oscillations, expressing exclusively the oscillations of the non-perturbed rotation of the rigid body. From this resolution, we obtain the Eulerian frequencies:

$\Omega_{f}=\sqrt{\frac{L^{2}-2 A H}{C(C-A)}}$

and

$\sigma=\Omega_{f} \sqrt{\alpha \beta}$

that give for Mercury the two periods $\Omega_{f}$ and $\sigma$ (where $L$ is the angular momentum in $O X Y Z, H$ the energy). $\alpha$ and $\beta$ are the dynamical coefficients of the body related to $A, B$ and $C$ by the following relations:

$\alpha=\frac{C-B}{A}$ and $\beta=\frac{C-A}{B}$. 

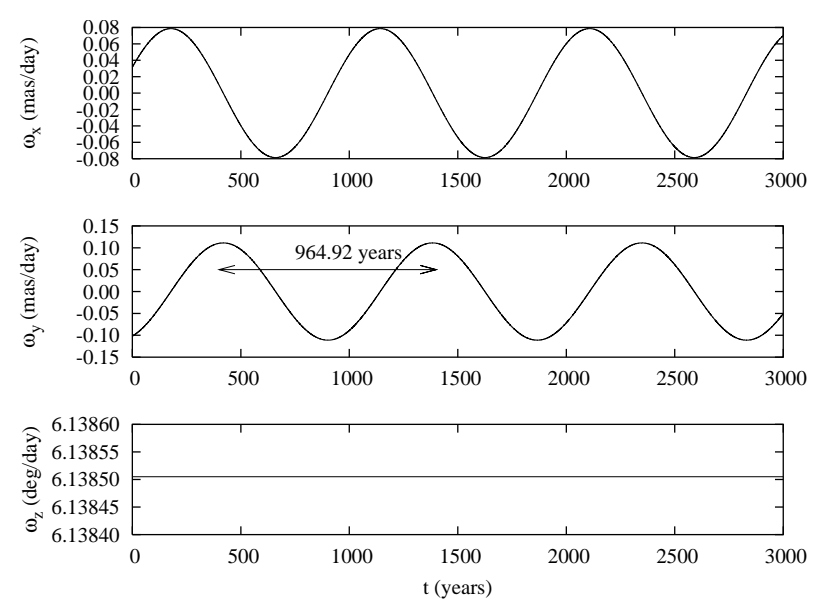

Fig. A.1. The free rotation of rigid Mercury in the components of its instantaneous rotation vector expressed in the body-fixed system $O x y z$ and plotted over 3000 yrs. Milliarseconds per day are on the vertical axis for $\psi$ and $\theta$ angles, degrees per day for $\varphi$, and years on the horizontal axes. The clearly visible period is the Euler period for Mercury: namely 964.92 yrs. The mean value of $\omega_{z}$ is $6.138 \mathrm{deg} / \mathrm{day}$.

With the previous initial conditions given in Table $1(\dot{\psi}=\dot{\theta}=$ $0)$, the free rotation of Mercury is reduced to an elementary rotation of the $\varphi$ angle only $\left(\Omega_{f}=\omega_{z}\right)$. Starting with the physical mean values of $\dot{\psi}, \dot{\theta}$, and $\eta_{0}$ evaluated in the present paper, $\Omega_{f}=58.646$ days and $\sigma=964.88$ yrs. The period of 58.646 days corresponds to the Hermean polar rotation while the one of 964.88 yrs means the global period of rotation of Mercury in $R^{3}$, which is equivalent to the Earth's classical Euler period, namely 305 days (Lambeck 1980). In the case of the Moon, this period is equal to $148.129 \mathrm{yrs}$.

Using the SONYR model reduced to the free rotation of Mercury, we obtain the components of the instantaneous rotation vector $\omega$ in the body-fixed system $O x y z$, as presented in Fig. A.1. $\omega_{z}$ is well found constant while the Euler period is equal to 964.92 yrs.

\section{References}

Anderson, J. D., Colombo, G., Esposito, P. B., Lau, E. L., \& Trager, G. B. 1987, Icarus, 71, 337

Anselin, A., \& Scoon, G. E. N. 2001, Planet. Space Sci., 49, 1409

Balogh, A., \& Giampieri, G. 2002, Rep. Prog. Phys., 65, 529

Béletski, V. 1986, in Essais sur le mouvement des corps cosmiques (Mir)

Bizouard, C., Schastok, J., Soffel, M., \& Souchay, J. 1992, in Journées 1992: Systèmes de référence spatio-temporels, ed. N. Capitaine, Observatoire de Paris

Bois, E. 1995, A\&A, 296, 850
Bois, E. 2000, C. R. Acad. Sci. Paris, t. 1, Série IV, 809

Bois, E., \& Journet, A. 1993, Celestial Mechanics and Dynamical Astronomy, 57, 295

Bois, E., \& Vokrouhlický, D. 1995, A\&A, 300, 559

Bois, E., \& Girard, J. F. 1999, Celestial Mechanics and Dynamical Astronomy, 93, 329

Bois, E., Wytrzyszczak, I., \& Journet, A. 1992, Celestial Mechanics and Dynamical Astronomy, 53, 185

Bois, E., Boudin, F., \& Journet, A. 1996, A\&A, 314, 989

Bois, E., Kiselea-Eggleton, L., Rambaux, N., \& Pilat-Lohinger, E. 2003, ApJ, submitted [astro-ph/0301528]

Borderies, N. 1978, Celestial Mechanics, 18, 295

Colombo, G. 1965, Nature, 208, 575

Colombo, G. 1966, AJ, 71, 891

Colombo, G., \& Shapiro, I. 1966, Smithsonian Astrophys. Obs. Spec. Rept., 188R, 296

Chirikov, B. V. 1979, Phys. Rep., 52, 263

Damour, T., Soffel, M., \& Xu, Ch. 1991, Phys. Rev. D, 43, 3273

Damour, T., Soffel, M., \& Xu, Ch. 1992, Phys. Rev. D, 45, 1017

Damour, T., Soffel, M., \& Xu, Ch. 1993, Phys. Rev. D, 47, 3124

Damour, T., Soffel, M., \& Xu, Ch. 1994, Phys. Rev. D, 49, 618

Ferrari, A. J., Sinclair, W. S., Sjogren, W. L., Williams, J. G., \& Yoder, C. F. 1980, Journal of Geophys. Res., 85, B7 3939

Fukushima, T. A\&A, 244, L11

Goldreich, P., \& Peale, S. 1966, AJ, 71, 425

Goldstein, H. 1981, in Classical Mechanics, ed. Columbia university

Jehn, R., \& Corral, C. 2003, Planet. Space Sci., submitted

Kaula, W. M. 1966, in Theory of satellite geodesy (Massachusetts: Blaisdell, Waltham)

Laskar, J., \& Robutel, P. 1993, Nature, 361, 608

Lambeck, K. 1980, in Earth's variable rotation: geophysical causes and consequences (Cambridge Univ. Press)

Landau, L., \& Lifchitz, E. 1986, Mécanique (Mir)

Milani, A., Vokrouhlický, D., \& Bonanno, C. 2001, Planet. Space Sci., 49,1579

Murray, C. D., \& Dermott, S. F. 2000, in The Solar System Dynamics (Cambridge)

Peale, S. J. 1969, AJ, 74, 483

Peale, S. J. 1972, Icarus, 17, 168

Peale, S. J. 1973, Reviews of Geophysics and Space Physics, 11, 767

Peale, S. J. 1988, in Mercury, ed. F. Vilas, C. Chapman, \& M. Matthews (Tucson: University of Arizona Press)

Peale, S. J. 1997, Lun. Planet. Sci. XXVIII

Pettengill, G. H., \& Dyce, R. B. 1965, Nature, 206, 1240

Schutz, B. E. 1981, Celestial Mechanics, 24, 173

Seidelmann, P. K., Abalakin, V. K., Bursa, M., et al. 2002, Celestial Mechanics and Dynamical Astronomy, 82, 83

Solomon, S. C., \& 20 colleagues 2001, Planet. Space Sci., 49, 1445

Standish, E. M. 1998, JPL IOM 312.F - 98 - 048

Wisdom, J., Peale, S. J., \& Mignard, F. 1984, Icarus, 58, 137

Wisdom, J. 1987, Icarus, 72, 241

Wu, X., Bender, P. L., \& Rosborough, G. W. 1997, J. Geophys. Res., 100,1515 